\title{
On Application Of A New Ant Colony Algorithm Model In Technology Foresight
}

\author{
Zhao LIU ${ }^{1, a}$, Mei ZHANG ${ }^{2, b}$, Xia XU ${ }^{3, c}$ \\ ${ }^{1}$ Department of Computer Science, Guangdong University of Technology, \\ Guangzhou, 510006, China \\ 2 Department of Computer Science, Guangdong University of Technology, \\ Guangzhou, 510006, China \\ ${ }^{3}$ Zhongkai University of Agriculture and Engineering, \\ Guangzhou, 510006, China \\ aemail: 190381200@qq.com, bemail:646054552@qq.com, cemail: Iz8797483@163.com
}

Keywords: Technology Foresight; Ant Colony Algorithm; Topology Network

\begin{abstract}
With the pace of development of science and technology continue to accelerate, the traditional technology foresight has been often limited by it's long cycle period and large expend. This paper presents a new model in a traditional method acting that --- Ant Colony Algorithm. Facing the basis problem, the idea is "Agent" on topology have two tendencies, which we define as "Convergence" and "Extinction". By interaction and updated coefficient, to find the best industry directions . In this basis, iterative technology foresight and Ant Colony model to build dimensional network model, and the process description of the algorithm and the convergence of the algorithm are expressed by mathematical formula.
\end{abstract}

\section{Introduction}

In the 21 st century, science and technology at an unprecedented pace, to the political, economic and social development and people's work and life have a profound change. Competition in the global economy and science and technology is heating; the study found that the risk of new product development and cost models and techniques, often by the developed countries, emerging disciplines, interdisciplinary and cross-sectoral cooperation projects guided. In order to accurately grasp the future development trend of science and technology and its impact on the economy, society and environment, identify priority areas of development, carry out technology foresight activities have become more and large enterprises a conscious decision to select [1]. Technology Foresight in the enterprise as a strategic management tool, is committed to scientific, technical, technological and economic forecasting in the whole of the next period of time, select those research projects of strategic importance by the method of measurement science, cutting-edge technology and general technology, allocation of resources, the use of market economic laws selection mode, to maximize social and economic benefits [2-5].

\section{Traditional methods and Technology Foresight Technology Foresight Research Status}

Delphi Method is the most commonly used and effective method in the research of technology foresight. Delphi method is a quantitative and a structured decision support technology, itself is in some quantitative information collection process, through multidimensional expert independent and repeatedly of subjective judgment (subjective judgment, but won a relatively objective information, advice and insights [4]. The essence of the Delphi method for a according to the subjective forecast method to forecast the technology, each of the steps are required to follow the scientific method, especially in dealing with a large number of questionnaire, itself is using the professional statistics and economics knowledge, which makes the system meter with the objective factors, illustrate the 
quantitative Research on the subject of qualitative research, the conclusions more scientific [1-7]. But due to the following reasons, making the Delphi law in the second industry to develop the limitations of industrial planning route very prominent:

1. need a lot of resources be devoted to investigation, (organized a team of experts) manpower and material resources (workload), making a number of small and medium-sized enterprises can not afford the cost of: as shown in Table 1, Japan only in a field opened a hundreds of subject, and the rate is not ideal.

2. the requirements of investigators and experts are very high, and can not avoid the negative impact of subjective initiative on the objective law.

3. the Delphi method itself is static, one-time predicted, the prediction model can not adapt to the situation of social science and technology development in the changing, figure 1 shows the technology forecasting in the cycle repeated instability [10]. Ant colony algorithm in after the optimization, the model of factor agent within a short period also multidirectional parallel activities and the continuous exchange of information and update the system parameters, the plan to gain technology roadmap intelligent planning model more close to the optimal solution.

Tab.1: The situation of Japanese Technology Foresight in 2011

\begin{tabular}{lcccc}
\hline Field & Evaluation task $(\mathrm{n})$ & Done (\%) & Partial Done (\%) & Not implemented (\%) \\
\hline Healthcare & 83 & 19 & 63 & 18 \\
Food and agriculture & 96 & 29 & 48 & 23 \\
Information science & 105 & 34 & 31 & 35 \\
Social development & 130 & 15 & 39 & 46 \\
Industry and resources & 174 & 29 & 26 & 45 \\
Total & 588 & $26($ avg $)$ & $38($ avg $)$ & 36 (avg) \\
\hline
\end{tabular}

Figure 1: Technology forecasting stability of each cycle

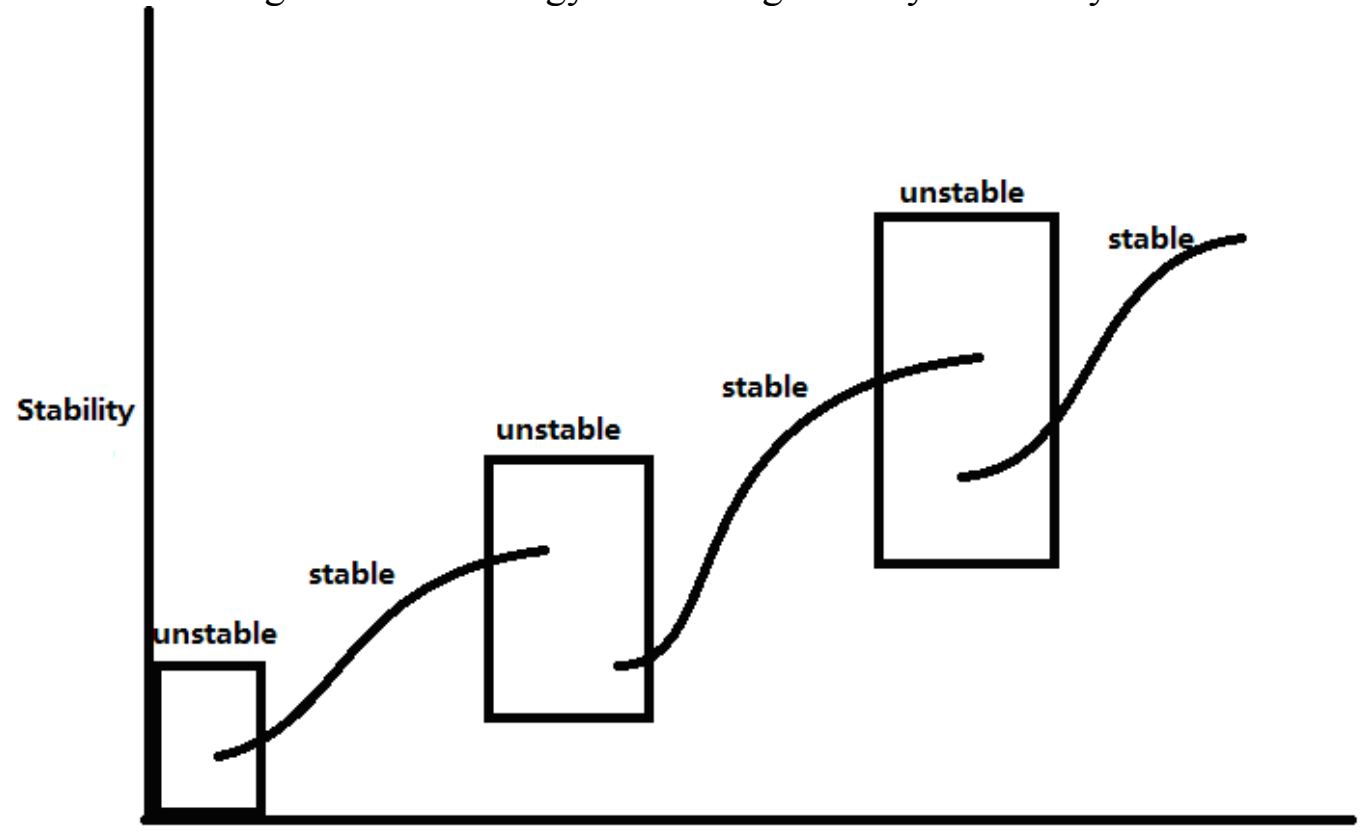

Cycle time

\section{The theoretical basis of the application of Ant Colony Theory in Technology Foresight}

By nature of ant colony foraging behavior inspired, Italy scientist M. Dorigo in the nineties of the last century through his doctoral dissertation "ant system optimization by a colony of cooperating agents proposed a used to find optimal solutions of probabilistic algorithms in the graph (or the network topology) and ant colony optimization (ACO). At present, the theory of ant colony as industry technology roadmap search research, mainly including pheromone transfer, autocatalytic, evaporation mechanism, data collection dynamic etc. methods in help enterprises 
develop industry roadmap, explore the law of science and technology development, to explore cutting-edge interdisciplinary research, technology research hot spot and on the potential of technology foresight and so on have a unique research value [11]. JE Moffitt pointed out that the information convergence, technology portfolio optimization and scientific development path optimization can be used as the means and methods of enterprise performance evaluation and development of science and technology. And ant colony algorithm by using ant colony foraging theory of technology products of development path analysis and processing, to predict the trend of the development of science and technology, indeed its industrial technology route, with is characterized for the future direction of development of science and technology planning in the short term, and the relative

Delphi Method of long period prediction has certain complementary time and improving traditional technology foresight the science and accuracy. At the same time, technology roadmapping development can image reveals Technology product evolution, development history, frontier and the whole knowledge system structure, reveal the dynamic development rules of domain knowledge, can effectively make up for the defects of the Delphi method, scenario analysis and other qualitative methods, to improve the accuracy and reliability of the development of industrial technology road mapping. [12]

\section{Ant colony algorithm for the prediction of the technology and basic concepts of ATF model}

Firstly, Tree of Technology Foresight ATF (Ant) is built to abstract the abstract data structure (Figure 2):

Figure 2: ATF model binary tree

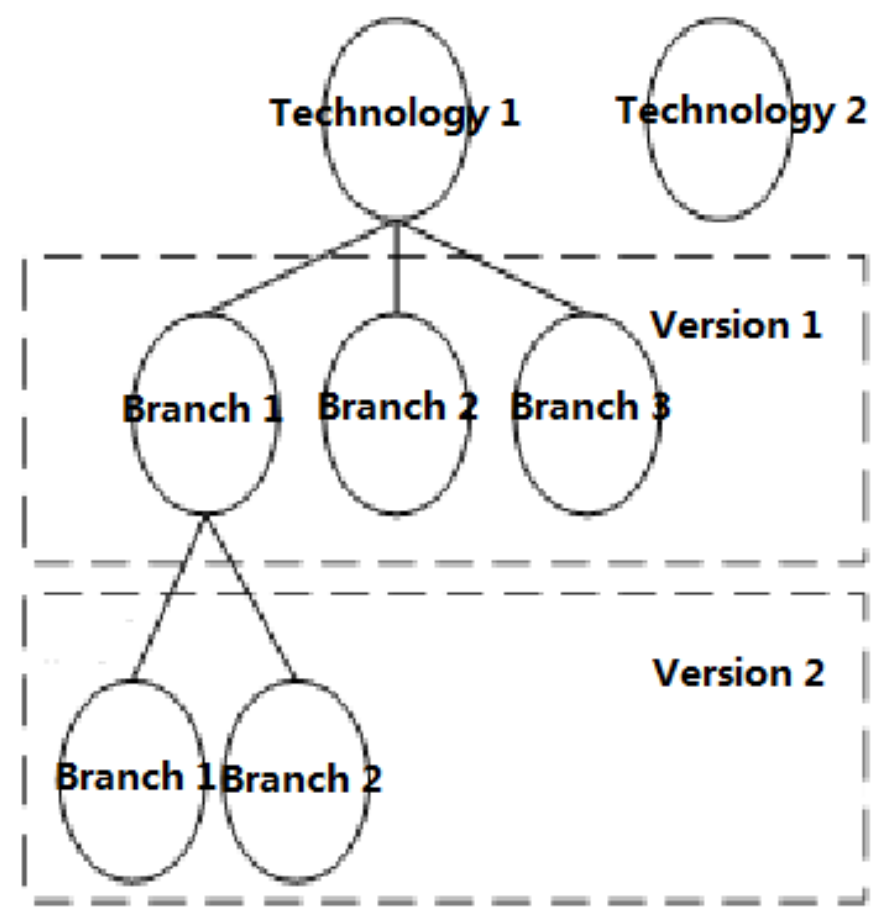

From Figure 2 it can be seen that when we put a technology roadmap (here we can understand a colony of ants foraging routes) design into a non AVL tree, each technique is the tree root ganglion (root); and each depth of the same parent sibling nodes is the version set $\mathrm{n}$; branch node $B_{11}, B_{12}, B_{13} \ldots B_{1 n}$ in us as brothers Siblings; from node $B_{1 n}$ to $B_{n n}$ path is defined as a specific technology roadmap.

If there is a path from $B_{1 n}$ to $B_{n n}$ in the reality, then $B_{1 n}$ is the ancestor of $B_{n n}$ and $B_{n n}$ is the descendants of $B_{1 n}$. If $B_{11}=B_{n n}$, then $B_{11}$ is the proper ancestor of $B_{n n}$ and $B_{11}$ is the proper descendant of $B_{n n} B_{11}$. 


\section{ATF Ant survival model}

ATF model ants living environment "extending a suitable path" behavior, the behavior of each artificial ant "agent" is very simple, when looking for him to adapt to the route of the extension, it is the route to the mobile; on the contrary, when the scan cycle to a certain number of times and "agent" not moving signs will automatically disappear [12]. So that the best of the best to know the best adaptive evolutionary path".

The ATF system of the ant Agent "feed" in the scope of activities for the two $\left\{B_{11} \ldots B_{1 n}\right\}$ $\times\left\{B_{11} \ldots B_{n 1}\right\}$ in network. In order not to let the brothers and descendants does not exist and appear in the calculation of the error and inconvenience, we put the topology network, the lower boundary and the left and right boundaries are connected.

The state will mark each ant in the agentij network $s_{i j}=\left\{x_{i j}, y_{i j}, \alpha_{i j}\right\}(1 \leq i, j \leq n)$. Among them, the number $n$ did not extinct; $x_{i j}$ and $y_{i j}$ as the plane coordinate; the degree of the disappearance of $\alpha_{i j}$ for agentij. $N\left(\right.$ agent $\left._{i j}\right)$ is defined as the eight brothers around and the descendants of the ancestors. Then the current adaptation of agent ij is F(agentij) [12]:

$$
F\left(\text { agent }_{i j}\right)=\left\{0, \frac{1}{9} \sqrt[2]{\sum_{\text {agent } \in N(\text { agentij }}\left(1-\frac{\mathrm{d}(\text { agent,agnet } \mathrm{tij}) \cdot \mathrm{d}\left(\text { agent }_{\mathrm{ij}}, \text { agent }\right)}{\mathrm{aij}}\right)^{2}}\right\}
$$

The $d$ (agent,agent $\left.t_{i j}\right)$ is the epsilon distance form agent $\in N$ (agentij) to agent $t_{i j}$, suggested in algorithm initialization phase pre processing calculated, the positioning of $\alpha_{i j}$ :

$$
\alpha_{i j}=\left\{\frac{1}{n} \sqrt[2]{\sum_{k=1}^{n} d\left(\text { agent }_{i 1}, \text { agent }_{i k}\right)^{2}}\right\} \times\left\{\frac{1}{n} \sqrt[2]{\sum_{k=1}^{n} d\left(\text { agent }_{j 1}, \text { agent }_{j k}\right)^{2}}\right\}
$$

The probability of an ant's demise in the environment is represented by $\mathrm{Pa}$ :

$$
P a\left(\text { agent }_{i j}\right)=\frac{e^{\beta \times F(\text { agentij })}}{\sum_{\text {agent } \in N(\text { agentij })} e^{\beta \times F(\text { agent })}}
$$

$\beta \in R^{+}$,called for the launch of the activation threshold; coefficient of extension:

$$
\Omega=\sum_{i=1}^{n} \min p_{a}\left(\text { agent }_{i j}\right)
$$

\section{Algorithm description and parameter setting}

Through the above description and the definition of parameters, the technical route of ATF industry convergence problem can be described as follows:

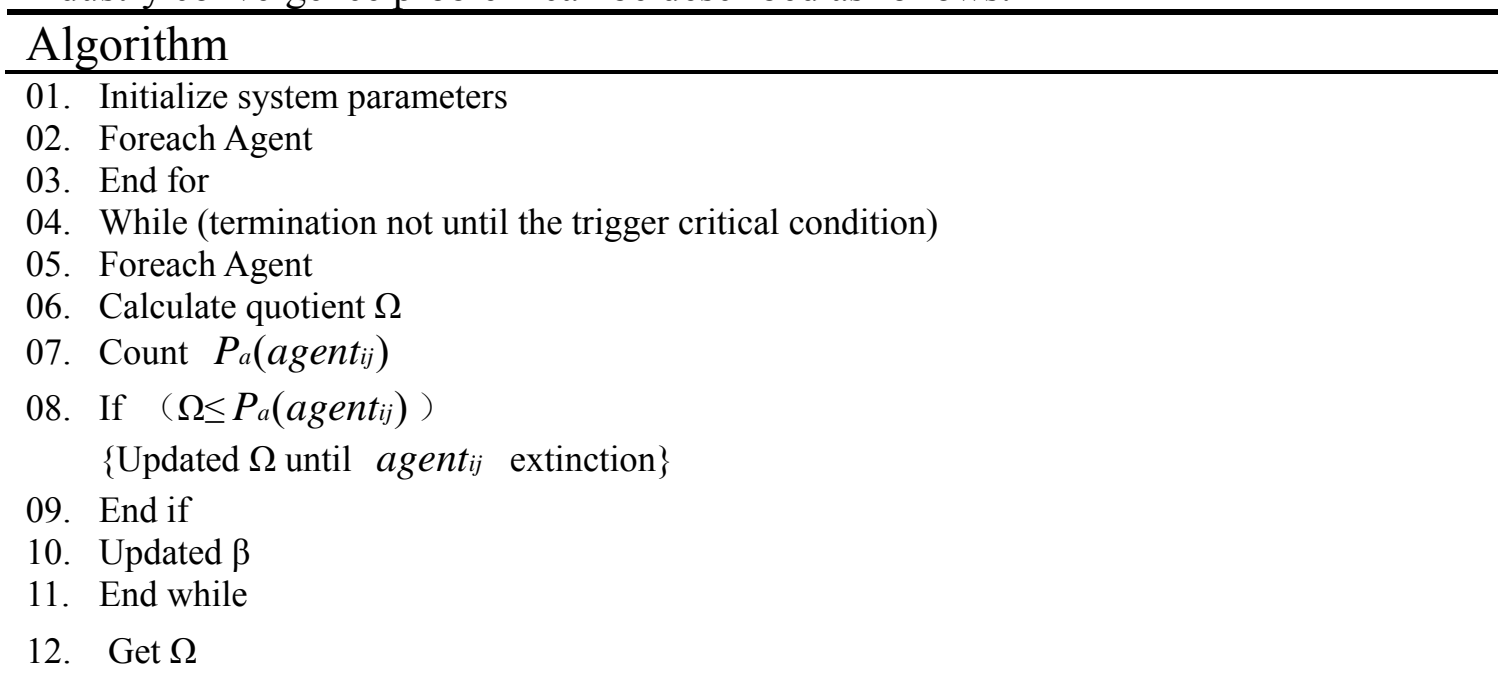

The initial value of the beta is constant. In the network system in the process of Agent in the process of beta update iteration cycle, the magnitude of the industry should be based on the actual 
situation.

\section{Conclusion}

In this paper, "the former technology foresight investment is too big" and "the possibility of the extension of the ant colony model" as the breakthrough point. A preliminary study of the combination of traditional technology and prediction and new ATF 2D network model, put forward a can achieve convergence of industrial technology route planning algorithm flow, put forward a new possibility and way for the future of small and medium enterprises technology forecast.

\section{Acknowledgement}

In this paper, the research was sponsored by the Natural Science Foundation of Guangdong Province (Project No. S2011040004281, S2013010014457).

\section{References}

[1] WANG Jin-bo. Technology Foresight: science and technology strategic planning and science and technology policy development [J]. China Soft Science, 2004, fifth (5): 62-66.

[2] PU Gen-xiang et al. On the basic assumption of the theory of Technology Foresight: the research of [J]. natural dialectics , 2006, 18 (7): 40-43.

[3] LI Guo-qiu,LONG Yi. Nearly a decade (2004 to 2013) International Technology Foresight research focus and Trend Analysis [J]. Document, Information \& Knowledge, 2014, 3 (3): 104-116.

[4] LI Guo-qiu,WANG Xiao-yun. Technology Foresight applied to predict the market feasibility analysis and implementation process [J]. Library Journal, 2014, 1 (1): 8-19.

[5] WANG Yin, XIE Jian-ying. An Adaptive ant colony optimization algorithm and simulation [J]. JOURNAL OF SYSTEM SIMULATION, 2002, 14(1):31-33

[6] Wang J F, Liu J H, Zhong Y F. A novel ant colony algorithm for assembly sequence planning[J]. International Journal of Advanced Manufacturing Technology, 2005, 25(11-12):1137-1143.

[7] Yang Z, Yu B, Cheng C. A Parallel Ant Colony Algorithm for Bus Network Optimization:[J]. Computer-aided civil and infrastructure engineering, 2007, 22(1):44-55.

[8] XU Xiao-hua,CHEN.Lin.An adaptive ant clustering algorithm [J]. Journal of Software , 2006, 9 (9): 1884-1889.

[9] Uğur A, Aydin D. An interactive simulation and analysis software for solving TSP using Ant Colony Optimization algorithms[J]. Advances in Engineering Software, 2009, 40(5):341-349.

[10] Kanama D. Development of technology foresight:integration of technology roadmapping and the Delphi method[M]//Technology Roadmapping for Strategy and Innovation. Springer Berlin Heidelberg, 2013: 151-171.

[11] Meissner D, Gokhberg L, Sokolov A. Science, Technology and Innovation Policy for the Future: Potentials and Limits of Foresight Studies[M]. Springer Science \& Business Media, 2013.

[12] GONG Hui,JIANG Ting,JIANG Gang-wu,et al. A convergence algorithm based on quaternion space resection global [J]. Acta Geodaetica et Cartographica Sinica, 2011, fifth (5): 639-645. 